\title{
Autobiographical Memory From Different Life Stages in Individuals With Obstructive Sleep Apnea
}

\author{
Neha Delhikar, ${ }^{1}$ Lucy Sommers, ${ }^{1,2}$ Genevieve Rayner, ${ }^{3,4}$ Rachel Schembri, ${ }^{2}$ Stephen R. Robinson, ${ }^{1,2}$ Sarah Wilson, ${ }^{4}$ AND \\ Melinda L. Jackson ${ }^{1,2}$ \\ ${ }^{1}$ School of Health and Biomedical Sciences, RMIT University, Victoria, Australia \\ ${ }^{2}$ Institute for Breathing and Sleep, Heidelberg, Victoria, Australia \\ ${ }^{3}$ Florey Institute of Neuroscience and Mental Health, Victoria, Australia \\ ${ }^{4}$ Melbourne School of Psychological Sciences, University of Melbourne, Victoria, Australia \\ (Received June 4, 2018; Final Revision October 29, 2018; Accepted November 5, 2018; First Published Online January 30, 2019)
}

\begin{abstract}
Objectives: Autobiographical memory dysfunction is a marker of vulnerability to depression. Patients with obstructive sleep apnea (OSA) experience high rates of depression and memory impairment, and autobiographical memory impairments have been observed compared to healthy controls; however, these groups were not age-matched. This study aimed to determine whether individuals with untreated OSA have impaired autobiographical memory when compared to age-matched controls, and to assess the quality of autobiographical memories from three broad time points. Methods: A total of 44 participants with OSA $(M$ age $=49.4 \pm 13.0)$ and 44 age-matched controls $(M$ age $=50.0 \pm 13.1)$ completed the Autobiographical Memory Interview (AMI) to assess semantic and episodic memories from three different life stages, and 44 OSA participants and 37 controls completed the Autobiographical Memory Test (AMT) to assess overgeneral memory recall (an inability to retrieve specific memories). Results: OSA participants had significantly poorer semantic recall of early adult life on the AMI $(p<.001)$, and more overgeneral autobiographical memories recalled on the AMT $(=.001)$, than controls. Poor semantic recall from early adult life was significantly correlated with more depressive symptoms $(p=0.006)$ and lower education $(p<0.02)$, while higher overgeneral memory recall was significantly associated with older age $(p=.001)$. Conclusions: A specific deficit in semantic autobiographical recall was observed in individuals with OSA. OSA patients recalled more overgeneral memories, suggesting that aspects of the sleep disorder affect their ability to recollect specific details of events from their life. These cognitive features of OSA may contribute to the high incidence of depression in this population. (JINS, 2019, 25, 266-274)
\end{abstract}

Keywords: Sleep, Depression, Semantic memory, Episodic memory, Polysomnography, Lifespan

\section{INTRODUCTION}

Obstructive sleep apnea (OSA) is a respiratory disorder characterized by complete or partial cessation of airflow through the upper airway multiple times during sleep, causing intermittent hypoxia and sleep disturbance. It is estimated to affect $1-4 \%$ of middle aged adults (Young et al., 1993), rising to $24-30 \%$ of the elderly (Bixler, Vgontzas, Lin, Tyson, \& Kales, 1998). Previous research has linked OSA to several neuropsychological impairments (Jackson, Howard, \& Barnes, 2011). Specifically, impairments in the immediate and delayed recall of verbal episodic memory (Kloepfer et al., 2009; Naëgelé et al., 2006; Twigg et al., 2010) suggest that

Correspondence and reprint requests to: Melinda L. Jackson, RMIT University, School of Health Sciences, P.O. Box 71, Building 201, Bundoora, VIC 3083, Australia. E-mail: melinda.jackson@ @rmit.edu.au those with OSA may be unable to effectively encode or consolidate information (Salorio, White, Duntley, Uhles \& Piccirillo, 2002; Wallace \& Bucks, 2013). A specific retrieval deficit of episodic memory, with intact maintenance and recognition, has also been reported in OSA, indicative of prefrontal dysfunction (Naëgelé et al., 2006). In addition, there is strong evidence that working memory deficits are common in patients with OSA, which may impact on the ability to conduct temporally-related cognitive processes, such as autobiographical memory (Sweet, Jerskey, \& Aloia, 2010).

These impairments can have significant implications in terms of declines in work productivity (Nena, Steiropoulos, Constantinidis, Perantoni, \& Tsara, 2010), increased risk of developing dementia (Chang et al., 2013), and mood problems (Jackson, Stough, et al., 2011; Naqvi, Wang, Glosier, 
\& Grunstein, 2014). Despite the known verbal episodic memory impairment in OSA, there has been little research into the integrity of autobiographic memory in particular, which is important to understand given its ecological validity as well as its links to depression.

Autobiographical memory is a record of an individual's experiences throughout their lifetime and can be characterized in terms of its specificity. Specific autobiographical memories are singular events localized to a particular place that span less than a day, such as participating in the high jump at a school sports carnival, or a sibling's birthday party at the zoo. Poorly specified or "overgeneral" autobiographical memories have been associated with developing and persistent depression (Williams \& Broadbent, 1986), and are considered a trait-like marker of vulnerability to the development and persistence of depression in the context of high life stress (Gibbs \& Rude, 2004; Sumner, Griffith \& Mineka, 2010).

While autobiographical memory has been explored extensively in terms of its nature, structure, and the way it is encoded, stored, and retrieved (Williams et al., 2007), research into autobiographical memory in individuals with OSA has been limited. Given that OSA is linked to verbal memory deficits as well as high rates of depression, it is worth exploring whether autobiographic memory is impaired in individuals with OSA, and whether this may underpin the high comorbidity of depression in OSA.

Of the limited research available, one recent study measured autobiographical memory in individuals with OSA, contrasting those with high versus low levels of self-reported depressive symptoms (Lee, Trinder, \& Jackson, 2016). Results showed that people with OSA recalled significantly fewer specific autobiographical memories compared to healthy controls. Contrary to expectations, however, there was no significant difference in autobiographical recall between OSA participants who were symptomatic or asymptomatic for depression, suggesting that impaired autobiographical memory recall was independent of mood state in this sample. A limitation of this study was that the sample of healthy controls was significantly younger than the OSA group, with sub-analysis of OSA participants aged 25-49 years showing no significant difference in their recall of specific autobiographical memories compared to agematched healthy controls. As such, further investigation is needed to determine whether an autobiographical memory deficit is present in a typical sample of individuals with OSA relative to age-matched controls.

For a more nuanced understanding of the potential impact of OSA on autobiographic memory function, these memories can be further classified as "episodic" or "semantic" in nature. Semantic autobiographical memory refers to one's personal knowledge of facts and concepts from their personal history (e.g., the names of one's school teachers), while episodic autobiographical memory refers to one's recollection of events or episodes from their past (e.g., first day of high school). Autobiographical memory is classified temporally, with episodic and semantic memories broadly clustered according to lifetime periods such as childhood, early adulthood, and recent life (Kopelman, Wilson, \& Baddeley, 1989).

Dissociations between semantic and episodic memory impairments have been observed in various neurological patient groups (Paul, Blanco, Hames, \& Beatty, 1997; Viskontas, Mcandrews, \& Moscovitch, 2000), in keeping with the partially distinct neurobiological substrates for semantic versus episodic memories. While both types of memory broadly recruit a large-scale bilateral brain network encompassing the anterior-posterior midline and lateral cortical regions (the "autobiographic memory network"; Rayner, Tailby, Wilson, \& Jackson, 2017), current evidence suggests that semantic memories specifically rely on the temporal poles and the lateral temporal neocortex (Irish \& Piquet, 2013), while episodic components of autobiographic memory are dependent on mesial temporal lobe structures, such as the entorhinal cortex and hippocampus (Levine, Turner, Tisserand, \& Hevenor, 2004). Providing a putative neurobiological substrate for the memory deficits seen in OSA, magnetic resonance imaging studies of people with OSA have reported a significant loss of gray matter from regions that overlap with the autobiographic memory network, including the left hippocampus (Canessa et al., 2011) and temporal gyrus (Morrell et al., 2010).

Some aspects of cognition are amenable to OSA treatments. Administration of continuous positive airway pressure (CPAP) therapy has been shown to improve some aspects of cognitive impairment exhibited by individuals with OSA, such as attention and visuospatial learning; however, some functions remain impaired or do not return back to the level of healthy controls, including executive function and working memory (Ferini-Strambi et al., 2003; Jackson, McEvoy, Banks, \& Barnes, 2018). This may indicate that there is a relationship between the OSA and cognitive dysfunction; however, with no definitive way of measuring the initial onset of OSA (Olaithe \& Bucks, 2013), attempting to estimate when cognitive impairments commenced relative to the disease onset is difficult. An exploration of autobiographical memory for events at particular time points in one's life in individuals with OSA could help to provide a better understanding of when autobiographical memory became impaired, and which memory processes are most affected.

The present study aimed to determine whether individuals with OSA have impaired autobiographical memory relative to age-matched controls, and to identify whether there is a particular life stage where any deficits seem to emerge. This study also aimed to examine relationships between impaired autobiographical memory, depression, and polysomnographic measures of OSA severity. It was hypothesized that OSA patients would display (1) more overgeneral autobiographical memories, (2) impaired episodic memory, and (3) have poorer memory for early adulthood and recent life (when they were most likely to have developed OSA). It was further hypothesized that poorer autobiographical memory performance would be associated with higher depression ratings and more severe OSA. 


\section{METHODS}

\section{Participants}

The study sample consisted of 44 individuals with untreated OSA ( $M$ age $=49.4 \pm 12.3$ years) aged between 20 and 69 years, and 44 age-matched healthy controls (within \pm 6 years of the OSA participants; $M$ age $=50.0 \pm 13.1$ years) aged between 22 and 69 years. Participants reported no previous history of psychiatric, neurological or medical conditions, or learning disabilities, and all were screened for clinical depression using the Structured Clinical Interview for Depression. Participants with OSA were recruited from the Austin Health sleep laboratory, and had recently undergone a standard overnight diagnostic sleep study and received a diagnosis of OSA (Apnea-Hypopnea Index $[\mathrm{AHI}] \geq 10$ events per hr; Berry et al., 2012). The average AHI was in the moderate to severe range (mean AHI $=51.2 \pm 20.8$ events $/ \mathrm{hr}$, range $=25.4-106.4$ ). Healthy controls were recruited from another study population, and were excluded if they selfreported having a sleep disorder. This research was approved by the Austin Health Human Research Ethics Committee. Written informed consent was acquired from all participants before undertaking the study. Participants were provided with a $\$ 50$ shopping voucher for their involvement in the study.

\section{Materials}

The Autobiographical Memory Interview (AMI) (Kopelman et al., 1989) is a semi-structured interview that assessed the recall of episodic and personal semantic autobiographical memories from three broad time periods: childhood (period before school, primary school, and high school years), early adult life (career, wedding, children, and meeting someone new in their twenties) and recent life (last Christmas and holiday/journey). The Autobiographical Incident Schedule recalls three personal episodes from each time period (e.g., a wedding), scored on their richness in detail and how precisely they are located in place and time using a three-point scale: three points for an episodic memory specific in time and place, two points for memories that were personal but overgeneral or occurred several times, one point for a vague personal memory (e.g., I liked playing chess), and zero points for no response or a memory based on a semantic memory (e.g., I lived in Bundoora). There was a maximum of nine points for each time period (total score maximum $=27$ ). Scores $\leq 12$ are associated with an amnestic syndrome while 13 to 15 are indicative of a probable amnestic syndrome.

The Personal Semantic Schedule recalls personally relevant facts (e.g., classmates' names). Responses were weighed on the level of detail retrieved (e.g., house number, street name, and suburb) with a maximum of 21 points for each time period (maximum $=63$ ). Scores $\leq 47$ are associated with an amnestic syndrome and those between 48 and 49 are indicative of a probable amnestic syndrome. Confabulation is usually not an issue on this task (e.g., Paul et al., 1997), particularly in samples of patients with no known frontal lobe syndrome (Kopelman et al., 1989). The AMI has previously demonstrated good inter-rater reliability (.85) (Strauss, Sherman, \& Spreen, 2006).

The Autobiographical Memory Test (AMT) was used to assess participants' ability to recall specific events from their life when prompted with a cue word (Williams \& Broadbent, 1986). Participants were presented with a cue word, and asked to retrieve a specific memory that the word reminded them of. This memory was to be a specific event that lasted less than a day, and occurred at a particular time and place. Cue words were five negatively valenced words (e.g., guilty, failure) and five positively valenced words (e.g., proud, happy). Memories were scored as "specific" (distinct time and place was recalled "the day I graduated"), "overgeneral" (event lasting longer than a day, encompassed many events or refers to a person or object "my mum"), or no response. For any response other than "specific," a prompt for clarification of a specific memory was given, and the clarified memory was scored accordingly. The number of overgeneral memories recalled was the primary outcome, with higher scores indicating poorer memory function. Good internal reliability estimates of .79 and .72 have been previously reported (Griffith, Kleim, Sumner, \& Ehlers, 2012; Griffith, Sumner, et al., 2012).

The Hospital and Anxiety Depression Scale (HADS) is a screening tool used to measure the severity of anxiety and depression (Zigmond \& Snaith, 1983). It is a self-report questionnaire with 14 items scored from 0 to 3 . Two subscales contain seven items on anxiety and depressive symptoms, respectively, with each subscale having a maximum score of 21 points. On both subscales, a score of less than seven is interpreted as normal, 8 to 10 as "borderline," and more than 11 corresponding to anxiety or depression. This scale is a valid measure of the severity of the underlying disease and has previously been validated in individuals with OSA (Law, Naughton, Dhar, Barton, \& Dabscheck, 2014).

The Epworth Sleepiness Scale (ESS) was used to assess an individual's general level of daytime sleepiness, and is frequently used in the OSA population (Johns, 1991). It is a selfadministered eight-item questionnaire about how easily the respondent would fall asleep in different situations. Items are scored from 0 to 3 with a maximum score of 24 . Higher scores indicate more sleepiness, with a score from 0 to 10 considered to be "normal" and more than 10 indicative of pathological sleepiness.

Polysomnography (PSG) was used to record overnight diagnostic sleep studies in participants with OSA. The PSG was analyzed using the 2012 American Academy of Sleep Medicine to analyze and calculate the sleep and respiratory indices (Berry et al., 2012). PSG data were gathered from the patients' medical records, and included the following OSA severity measures: AHI; arousal index (number of electroencephalogram arousals per hour of sleep); $4 \%$ oxygen desaturation index (ODI; total amount of sleep time spent with a $4 \%$ blood oxygen desaturation); and sleep measures: total sleep time; sleep efficiency; percentage of rapid eye 
movement (REM) sleep across the night (\%REM) and percentage of non-REM (NREM) sleep across the night (\%NREM).

\section{Procedure}

Individuals with OSA were invited to participate in the study after their clinical diagnostic sleep study at Austin Health and confirmation of a diagnosis of OSA. Written consent to participate was signed before participation, and demographic data were collected from all participants at enrolment. On a separate visit, participants with OSA attended Austin Health to complete the memory tests. The AMI and the AMT were administered in the same order for all participants in a quiet room, and scored as described previously (Rayner, Jackson, \& Wilson, 2016). For scoring purposes, the AMT was recorded in audio using the Voice Memos application on a smartphone. An independent rater blind to group rescored $20 \%$ of the AMT and AMI responses. The intraclass correlation coefficient for the AMT was .99, and for the AMI was 95 .

Data from the control participants were collected through another study. These participants were taken from a subgroup of healthy individuals who were parents or carers of individuals attending hospital appointments, and completed the AMI, AMT, and HADS with a researcher during their visit. Controls were selected if they were within 6 years of age to each OSA participant enrolled in the study. The data from this healthy cohort have been published previously (Allebone, Rayner, Siveges, \& Wilson, 2015; Rayner, Jackson \& Wilson, 2015, 2016a).

\section{Statistical Analyses}

Analyses were run using IBM SPSS 24.0 (IBM Corp., Armonk, NY). Seven participants in the control group did not complete the AMT, leaving 37 in the final analysis for this measure. In addition, seven OSA participants did not report their years of education. The differences in group characteristics (age, years of education, and HADS scores) were examined using independent samples $t$ tests. Analyses of covariance were then conducted to compare specific and overgeneral memory recall on the AMT controlling for age, and semantic and episodic recall from the three time periods (childhood, early adult, and recent life) on the AMI, between individuals with OSA and healthy controls, controlling for age, years of education, and depressive symptoms (HADS-D).

Using the method by Brittlebank, Scott, Mark, Williams, and Ferrier (1993), the median AMT score for the control group was used to classify participants as having "high" and "low" overgeneral memory. Independent samples $t$ tests were conducted on these variable to examine difference between individuals with OSA with high (HADS-D $\geq 8 ; \mathrm{N}=19)$ and low (HADS-D < 8; $\mathrm{N}=25$ ) depressive symptoms. Pearson's correlations were conducted for those outcomes that were significantly impaired in the OSA group, to examine whether impaired memory performance was associated with demographic variables (age, years of education, body mass index [BMI]) depression (HADS-D), and sleep measures for the OSA group (ESS, AHI, arousal index, \%REM, \%NREM, total sleep time, sleep efficiency, and 4\% ODI). Statistical significance set at $p<.05$ (two-tailed) using an false discovery rate of 0.01 for all tests to correct for multiple comparisons.

\section{RESULTS}

Table 1 displays the demographic data from both groups. There was no significant difference in age between the OSA group and the control group $(\mathrm{t}(86)=-.22 ; p=.82)$. Participants with OSA had significantly higher depression $(t(86)=5.63 ; p<0.001)$ and anxiety $(\mathrm{t}(86)=2.15 ; p=.04)$ scores compared to controls, and had higher rates of a selfreported history of clinical depression, and current psychotropic drug use. There was a significantly higher proportion of males in the OSA group $\left(\chi^{2}=18.3 ; p<.001\right)$, reflecting the gender disparity in presentation of OSA in the wider community, however, there were no significant gender differences for any of the memory measures ( $p$ 's >.14).

Table 1. Demographic variables for OSA and control participants

\begin{tabular}{lcc}
\hline \hline & OSA & Controls \\
\hline$N$ & 44 & 44 \\
Females/males*** & $14 / 30$ & $34 / 10$ \\
Age, years & $49.4(13.0)$ & $50.0(13.1)$ \\
Age, range & $20-69$ & $22-69$ \\
BMI & $36.06(8.35)$ & \\
Years of education & $12.9(3.4)$ & $13.5(3.2)$ \\
Type of education & & \\
Primary & $2.5 \%$ & $0 \%$ \\
$\quad$ High school & $40.0 \%$ & $47.7 \%$ \\
Trade & $17.5 \%$ & $9.1 \%$ \\
University & $40.0 \%$ & $43.2 \%$ \\
AHI & $51.2(20.8)$ & - \\
Arousal Index & $34.4(16.7)$ & - \\
ODI $4 \%$ & $31.4(22.6)$ & - \\
Self-reported history of depression & $25.0 \%$ & $9.1 \%$ \\
Current psychotropic medication & $22.7 \%$ & $7.1 \%$ \\
ESS & $10.9(5.6)$ & - \\
HADS, anxiety* & $7.7(4.3)$ & $5.6(4.4)$ \\
No anxiety & $47.7 \%$ & $70.5 \%$ \\
Mild anxiety & $36.4 \%$ & $13.6 \%$ \\
Clinical anxiety & $15.9 \%$ & $15.9 \%$ \\
HADS, depression*** & $6.9(3.4)$ & $3.0(3.2)$ \\
No depression & $56.8 \%$ & $88.6 \%$ \\
Mild depression & $27.3 \%$ & $6.9 \%$ \\
Clinical depression & $15.9 \%$ & $4.5 \%$ \\
\hline \hline
\end{tabular}

Note. Values represent mean $(S D)$ unless otherwise stated.

$* * * p \leq 0.001$.

$* p \leq 0.05$. 
There was a similar level of education between groups $(\mathrm{t}(73)=-0.58 ; p=.57)$.

\section{Autobiographical Memory Test}

OSA participants recalled significantly more overgeneral memories compared to controls, indicating poorer autobiographic memory function $(F(2,81)=13.36 ; p<.001$; $\eta^{2}=0.26$; Table 2). Within the OSA group, $52.3 \%$ were classified as having high overgeneral memory ( $\geq 5$ overgeneral memories recalled) compared to $18.9 \%$ of the controls. Comparison of OSA participants with high and low depressive symptoms revealed no significant difference in the number of overgeneral memories recalled on the AMT $(p=.41)$.

\section{Autobiographical Memory Interview}

\section{Personal semantic memory schedule}

There was a trend for OSA participants having lower semantic autobiographical recall when compared to controls $(p=0.058)$. When examining different lifespan periods, participants with OSA specifically had significantly lower semantic recall for memories from the early adult lifetime period than healthy controls $(F(1,75)=7.54 ; p=0.008$; $\left.\eta^{2}=0.10\right)$. There was no significant difference in semantic autobiographical memory recall between individuals with OSA and controls from the childhood lifetime period $(p=.27)$ or recent life $(p=.92)$.

\section{Autobiographical incidents schedule}

There was no significant difference in total episodic memory recall between the OSA group and controls $(p=.11)$, nor were there any significant differences in episodic autobiographical memory recall between individuals with OSA and controls from the childhood $(p=.94)$ or early adult lifetime period $(p=.56)$. OSA participants had significantly

Table 2. AMI and AMT overgenerality scores for the OSA and control groups

\begin{tabular}{lrr}
\hline \hline & OSA & Controls \\
\hline AMI semantic total score $*$ & $54.85(4.07)$ & $57.15(4.14)$ \\
AMI sematic childhood & $17.74(2.57)$ & $18.36(2.60)$ \\
AMI sematic early adulthood $* * *$ & $18.42(2.28)$ & $19.86(1.44)$ \\
AMI sematic recent life & $18.74(1.76)$ & $18.92(1.51)$ \\
AMI episodic total score & $20.96(3.29)$ & $19.41(3.98)$ \\
AMI episodic childhood & $6.73(1.62)$ & $6.64(1.63)$ \\
AMI episodic early adulthood & $6.63(1.46)$ & $6.48(1.77)$ \\
AMI episodic recent life $* * *$ & $7.61(1.30)$ & $6.30(1.72)$ \\
AMT overgeneral $* * *$ & $4.93(2.68)$ & $3.16(1.74)$ \\
AMT specific & $5.93(2.55)$ & $6.05(1.96)$ \\
\hline \hline
\end{tabular}

Note. Values represent mean $(S D) . N=44$ for OSA and $N=44$ for controls, except $N=37$ for the control group AMT results.

$* * * p \leq 0.001$.

$* p \leq 0.05$. higher episodic autobiographical memory recall for the recent lifetime period when compared to controls $(F(1,75)=9.73$; $\left.p=.003 ; \eta^{2}=0.12\right)$.

Comparison of OSA participants with high and low depressive symptoms revealed no significant difference in total personal semantic $(p=.41)$ or autobiographical $(p=.46)$ memories recalled on the AMI.

\section{Association Between Autobiographical Memory Function and Demographic, Sleep, and Mood Variables}

Across both groups, HADS depression score was significantly associated with poorer early adulthood semantic memory recall $(\mathrm{r}=-0.29 ; p=.006)$, and higher number of years of education was significantly associated with better autobiographical memory recall from early adulthood $(\mathrm{r}=0.38 ; p=.001$; Figure 1$)$. Older age was significantly associated with higher overgeneral memory recall $(\mathrm{r}=0.38$; $p \leq .001)$. Within the OSA group, \%REM $(\mathrm{r}=-0.42$; $p=.005)$ and $\%$ NREM $(\mathrm{r}=0.43 ; p=.004)$ were significantly associated with recent life autobiographical memory. No relationship between autobiographical memory recall and BMI, or objective or subjective sleep measures reached statistical significance.

\section{DISCUSSION}

The present study investigated whether autobiographical memory was impaired in individuals with OSA when compared to age- and education-matched healthy controls, and whether the recall of autobiographical memories from different stages of life was differentially affected. This group of treatment-naïve, moderate-severe OSA participants had higher overgeneral memory recall and poorer semantic autobiographical memory recall from the early adult lifetime period when compared to age-matched healthy controls. Individuals with OSA also had higher episodic autobiographical memory recall from the recent lifetime period compared to healthy controls. Across the whole sample, increasing age was associated with a higher number of overgeneral autobiographical memories recalled, and higher depression scores were associated with worse semantic memory.

In line with our previous research (Lee et al., 2016), the present study found impairments on the AMT in individuals with OSA compared to controls, with more overgeneral memories recalled by those with OSA. In the study by Lee et al. (2016), the OSA group were significantly older than the controls; a limitation overcome here by comparing the OSA participants to age-matched controls. This finding supports previous studies that have reported verbal episodic memory impairments in OSA patients (Twigg et al., 2010; Wallace \& Bucks, 2013). In support of the study by Lee et al. (2016), no difference in the number of overgeneral memories between those reporting high and low depressive symptoms was observed. 

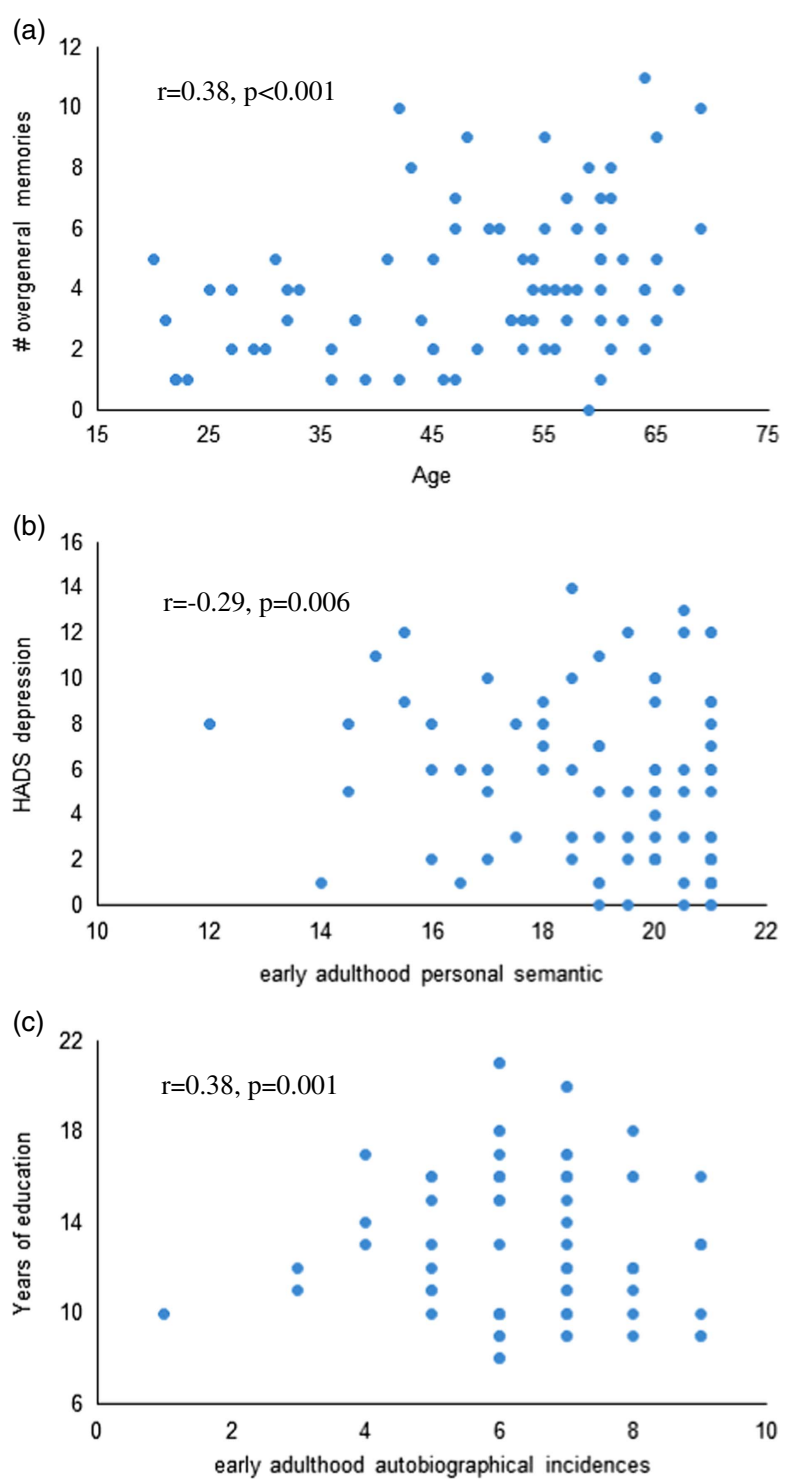

Fig. 1. Scatterplots of the correlations between (a) age and overgeneral memory recall on the AMT, (b) HADS depression and early adulthood personal semantic memory, and (c) years of education and early adulthood autobiographical incidences across both groups.

We did not find support for our hypothesis of impairment in episodic (autobiographical) memory recall on the AMI, with impairments in overall semantic, but not episodic, memory observed in the patient group. This finding is in line with previous reports of impaired semantic memory and intact episodic memory in patients with multiple sclerosis (Paul et al., 1997). The standard consolidation model proposes that all memories, including semantic autobiographical memories, are indexed and encoded in the hippocampal complex of the medial temporal lobe. Structural neuroimaging studies have found reduced brain volumes in these regions in OSA patients (Morrell, et al., 2003), which is likely due to chronic intermittent hypoxia.

There is a large body of literature implicating severe hypoxia in neonates in impaired encoding of new episodic memories, while semantic memory remains relatively intact (e.g., Cooper et al., 2015). On the contrary, the current study found impaired semantic memory in the OSA group, while episodic autobiographical memory was preserved. Thus, intermittent hypoxia sustained by adult OSA patients over months or years before attending the sleep laboratory may have differential effects on hippocampal functioning and the downstream memory processes compared to more severe hypoxia sustained in early life.

Sleep fragmentation in OSA has also been shown to have adverse effects on cognitive functioning (Naëgelé et al., 2006). The consolidation of semantic autobiographical memory is dependent on non-REM and REM sleep processes (Horton \& Malinowski, 2015). This sleep-dependent consolidation may be limited in individuals with OSA due to their poor sleep quality and fragmented sleep architecture (Guo, Igue, Malhotra, Stickgold, \& Djonlagic, 2013; Horton \& Malinowski, 2015), with inefficient or disrupted consolidation of sleep dependent memories providing a potential explanation for why semantic autobiographical memory is impaired in individuals with OSA. Both higher \%REM and lower \%NREM were associated with poorer recall of autobiographical (but not semantic) memories from recent life in the current study. However, why this impairment was especially accentuated in the early adulthood period is unclear.

It is possible that for some participants, early adulthood may have been the period of their life when they developed OSA symptoms, with the commencement of significant and persistent sleep disruption impairing the laying down and retention of memories from that time. Alternatively, this impairment may reflect degradation of remote memories that were initially successfully encoded, or a working memory deficit, impairing in the ability to successfully retrieve these more remote memories (Sweet et al., 2010).

Better episodic autobiographical memory recall from the recent lifetime periods in individuals with OSA is an unexpected finding. It contradicts the known impairments in cognition and memory in this population (Wallace \& Bucks, 2013), and the episodic memory impairment seen on the AMT. It is possible that the OSA patients were able to perform better than our healthy control group due to the circumstances surrounding their recent events (their hospital visit in particular), where there was more novelty and salience which may have facilitated encoding.

Of note, the performance of the healthy control group in this study ( $\mathrm{M}=6.3$ points) was below the acceptable range (7-9 points) for recent autobiographical memory recall compared to other normative samples; therefore, a more accurate interpretation of this finding may be that the performance of the OSA group is consistent with that of normative samples, rather than being superior. A limitation of the current study was that the presence of a sleep disorder in the control group was ruled out by self-report; therefore, it is possible that some of the control participants had undiagnosed sleep disordered breathing.

Across all participants, correlational analysis found that older age was associated with more overgeneral memory recall. Moreover, poorer total semantic memory recall from 
the early adulthood stage was associated with higher current depression scores, and lower education was associated with poorer autobiographical memory recall. These factors have all been previously demonstrated to have an influence on memory function in the healthy population (De Wit et al., 2016; Gibbs \& Rude, 2004; Rubin \& Schulkind, 1997).

In both psychiatric and non-clinical populations, overgenerality is strongly associated with the development and persistence of depressive symptoms. Accordingly, in the current study overgenerality was associated with higher HADS depression scores across the whole sample, with the OSA group having a higher proportion of participants with clinically significant HADS depression scores compared to controls ( $15.9 \%$ vs. $4.5 \%$, respectively), as well as $25 \%$ with a self-reported history of clinical depression. It has previously been proposed that abnormal functioning of the autobiographic memory network is a key biological signature of depression that correlates with the biased self-related processing characteristics of the disorder (Rayner, Jackson, \& Wilson, 2016b). This raises the question as to whether dysfunction of the autobiographic memory network gives rise to both depressive symptoms and autobiographical memory deficits in OSA patients; that is, their correlation stems from a shared neurobiological mechanism. This intriguing question should be followed up in future neuroimaging research.

The results of this study raise the question of whether the memory deficits in OSA stem from encoding problems or retrieval issues. Previous reports of reduced hippocampal volumes in OSA (e.g., Morrell et al., 2003) lend weight to encoding problems; however, functional imaging studies have implicated the default-mode network (Sweet et al., 2010; Khazaie et al., 2017), which is crucial for retrieval of autobiographical memories. It is also plausible that autobiographical memory deficits in our patient group were present before the onset of OSA. Due to the cross-sectional nature of this study, we cannot determine the direction of the effect.

The strength of the present study lies in the study design, which used age-matched controls and examined two related, but dissociable, aspects of autobiographical memory function. The AMT and AMI used in this study provide a unique understanding of memory processing in OSA, as unlike in traditional neuropsychological assessments of memory function, the memories recalled from these tests relate to salient knowledge from one's past, that is, information that the participants would have once known. Thus, any deficits must reflect impairments in remote memory.

An important next step will be to determine whether the treatment of OSA with CPAP helps to counter the impairments in autobiographical memory, to better understand the mechanisms of OSA on autobiographical memory. Previous studies have shown that autobiographical memory function may be a useful predictor of recovery of depressive symptoms after CPAP treatment in individuals with OSA (Svaldi \& Mackinger, 2003). Whether or not these impairments improve after successful treatment of OSA has not been examined, and may help to elucidate whether the deficits occurred during the encoding/consolidation of the memories and thus cannot be restored, or if memory improves with treatment, suggesting issues with retrieval. These outcomes will allow us to understand the role of untreated OSA on memory processing: whether it prevents the encoding and consolidation of memories of one's life, or if it inhibits the effective recollection and retrieval of these events.

This study demonstrated a deficit in semantic autobiographical memory recall and in the ability to retrieve specific memories from one's past in individuals with untreated OSA. These results point toward the likelihood that OSA impairs the capacity to either encode or consolidate semantic autobiographical memories, leading to difficulties with the recall of specific details from the past. Poor autobiographical memory is a risk factor for developing persistent depression, which is a significant disease burden in the OSA population. Whether this aspect of memory can be restored with the treatment of OSA, and how this impacts on patient mood, remains to be elucidated.

\section{ACKNOWLEDGMENTS}

M.L.J. was supported by a National Health and Medical Research Council of Australia Early Career Fellowship (APP1036292). This study was funded by an Institute for Breathing and Sleep research grant. The authors thank Prof. Williams for providing access to the Autobiographical Memory Test. The authors have no conflicts of interest to disclose. M.J., R.S., and S.R. contributed to the study conception and design. N.D., L.S., and G.R. collected the data and N.D. performed the analysis. M.J., G.R., S.W., and S.R. contributed to the interpretation of the data. All authors contributed to the drafting of the manuscript.

\section{REFERENCES}

Allebone, J., Rayner, G., Siveges, B., \& Wilson, S.J. (2015). Selfidentity and autobiographic memory in epilepsy. Epilepsia, 56, 1982-1991.

Berry, R.B., Budhiraja, R., Gottlieb, D.J., Gozal, D., Iber, C., Kapur, V.K., . . . Tangredi, M.M. (2012). Rules for scoring respiratory events in sleep: Update of the 2007 AASM Manual for the Scoring of Sleep and Associated Events. Journal of Clinical Sleep Medicine, 8(5), 597-619.

Bixler, E.O., Vgontzas, A.N., Lin, T., Tyson, K., \& Kales, A. (1998). Effects of age on sleep apnea in men: I. Prevalence and severity. American Journal of Respiratory and Critical Care Medicine, 157, 144-148.

Brittlebank, A.D., Scott, J., Mark, J., Williams, G., \& Ferrier, I.N. (1993). Autobiographical memory in depression: State or trait marker? The British Journal of Psychiatry, 162(1), 118-121.

Canessa, N., Castronovo, V., Cappa, S., Aloia, M., Marelli, S., \& Falini, A., . . . Ferini-Strambi, L. (2011). Obstructive sleep apnea: Brain structural changes and neurocognitive function before and after treatment. American Journal of Respiratory and Critical Care Medicine, 183(10), 1419-1426.

Chang, W., Liu, M., Chang, W., Yang, A.C., Ku, Y.-C., Pai, J., . . . Tsai, S. (2013). Sleep Apnea and the risk of dementia: A population-based 5-Year follow-up study in Taiwan. PLoS One, 8 (10), e78655. doi:10.1371/journal.pone.0078655 
Cooper, J., Gadian, D.G., Jentschke1, S., Goldman, A., Munoz, M., Pitts, G., ...Vargha-Khadem, F. (2015). Neonatal hypoxia, hippocampal atrophy, and memory impairment: Evidence of a causal sequence. Cerebral Cortex, 25, 1469-1476.

De Wit, L., Kirton, J., O’Shea, D., Szymkowicz, S., McLaren, M., \& Dotson, V. (2016). Effects of body mass index and education on verbal and nonverbal memory. Neuropsychology, Development, and Cognition. Section B, Aging, Neuropsychology and Cognition, 24, 256-263. http://dx.doi.org/10.1080/13825585.2016. 1194366

Ferini-Strambi, L., Baietto, C., Di Gioia, M., Castaldi, P., Castronovo, C., Zucconi, M., \& Cappa, S. (2003). Cognitive dysfunction in patients with obstructive sleep apnea (OSA): Partial reversibility after continuous positive airway pressure (CPAP). Brain Research Bulletin, 61(1), 87-92.

Gibbs, B., \& Rude, S. (2004). Over general autobiographical memory as depression vulnerability. Cognitive Therapy And Research, 28(4), 511-526. http://dx.doi.org/10.1023/b: cotr.0000045561.72997.7

Griffith, J.W., Kleim, B., Sumner, J.A., \& Ehlers, A. (2012). Factor structure of the autobiographical memory test in recent trauma survivors. Psychological Assessment, 24(3), 640-646.

Griffith, J.W., Sumner, J.A., Raes, F., Barnhofer, T., Debeer, E., \& Hermans, D. (2012). Current psychometric and methodological issues in the measurement of overgeneral autobiographical memory. Journal of Behaviour Therapy And Experimental Psychiatry, 43(1), S21-S31.

Guo, M., Igue, M., Malhotra, A., Stickgold, R., \& Djonlagic, I. (2013). The effect of obstructive sleep apnea on declarative memory consolidation. Sleep Medicine, 14, e33-e34. http://dx. doi.org/10.1016/j.sleep.2013.11.041

Horton, C., \& Malinowski, J. (2015). Autobiographical memory and hyperassociativity in the dreaming brain: Implications for memory consolidation in sleep. Frontiers in Psychology, 6, 874.

Irish, M., \& Piguet, O. (2013). The pivotal role of semantic memory in remembering the past and imagining the future. Frontiers in Behavioral Neuroscience, 7(27), 1-7.

Jackson, M.L., Howard, M.E., \& Barnes, M. (2011). Cognition and daytime functioning in sleep-related breathing disorders. Progress in Brain Research, 190, 53-68.

Jackson, M.L., McEvoy, D., Banks, S., Barnes, M. (2018). Neurobehavioral impairment and CPAP treatment response in mild-moderate obstructive sleep apnea. Journal of Clinical Sleep Medicine, 14(1), 47-56.

Jackson, M.L., Stough, C., Howard, M.E., Spong, J., Downey, L.A., \& Thompson, B. (2011). The contribution of fatigue and sleepiness to depression in patients attending the sleep laboratory for evaluation of obstructive sleep apnea. Sleep and Breathing, 15 (3), 439-445.

Johns, M. (1991). A new method for measuring daytime sleepiness: The Epworth sleepiness scale. Sleep, 14(6), 540-545.

Khazaie, H., Veronese, M., Noori, K., Emamian, F., Zarei, M., Ashkan, K., . . Rosenzweig, I. (2017). Functional reorganization in obstructive sleep apnoea and insomnia: A systematic review of the resting-state fMRI. Neuroscience and Biobehavioral Reviews, 77, 219-231.

Kloepfer, C., Riemann, D., Nofzinger, E., Feige, B., Unterrainer, J., O'Hara, R., . . Nissen, C. (2009). Memory before and after sleep in patients with moderate obstructive sleep apnoea. Journal of Clinical Sleep Medicine, 5(6), 540-548.
Kopelman, M., Wilson, B., \& Baddeley, A. (1989). The autobiographical memory interview: A new assessment of autobiographical and personal semantic memory in amnesic patients. Journal of Clinical and Experimental Neuropsychology, 11(5), 724-744.

Law, M., Naughton, M., Dhar, A., Barton, D., \& Dabscheck, E. (2014). Validation of two depression screening instruments in a sleep disorders clinic. Journal of Clinical Sleep Medicine, 10(6), 683-688. http://dx.doi.org/10.5664/jcsm.3802

Lee, V., Trinder, J., \& Jackson, M.L. (2016). Autobiographical memory impairment in obstructive sleep apnoea patients with and without depressive symptoms. Journal of Sleep Research, 25(5), 605-611.

Levine, B., Turner, G.R., Tisserand, D., \& Hevenor, S.J. (2004). The functional neuroanatomy of episodic and semantic autobiographical remembering: A prospective functional MRI study. Journal of Cognitive Neuroscience, 16, 1633-1646.

Morrell, M.J., McRobbie, D.W., Quest, R.A., Cummin, A.R.C., Ghiassi, R., \& Corfield, D.R. (2003). Changes in brain morphology associated with obstructive sleep apnea. Sleep Medicine, 4(5), 451-454.

Morrell, M.J., Jackson, M.L., Twigg, G.L., Ghiassi, R., McRobbie, D.W., Quest, R.A., . . . Corfield, D. (2010). Changes in brain morphology in patients with obstructive sleep apnoea. Thorax, $65,908-914$.

Naëgelé, B., Launois, S., Mazza, S., Feuerstein, C., Pépin, J., \& Lévy, P. (2006). Which memory processes are affected in sleep apnoea? An evaluation of 3 types of memory. Sleep, 29(4), 533544.

Naqvi, H.A., Wang, D., Glozier, N., \& Grunstein, R.R. (2014). Sleep-disordered breathing and psychiatric disorders. Current Psychiatry Reports, 16, 519.

Nena, E., Steiropoulos, P., Constantinidis, T., Perantoni, E., \& Tsara, V. (2010). Work productivity in obstructive sleep apnea patients. Journal of Occupational \& Environmental Medicine, 52(6), 622-625. doi:10.1097/jom.0b013e3181e12b05

Olaithe, M., \& Bucks, R. (2013). Executive dysfunction in OSA before and after treatment: A meta-analysis. Sleep, 36(9), 1297-1305.

Paul, R., Blanco, C., Hames, K., \& Beatty, W. (1997). Autobiographical memory in multiple sclerosis. Journal of the International Neuropsychological Society, 3(3), 246-251.

Rayner, G., Jackson, G.D., \& Wilson, S.J. (2015). Behavioural profiles in frontal lobe epilepsy: Autobiographic memory versus mood impairment. Epilepsia, 56, 225-233.

Rayner, G., Jackson, G.D. \& Wilson, S.J. (2016a). Mechanisms of memory impairment in epilepsy depend on age at disease onset. Neurology, 87, 1642-1649.

Rayner, G., Jackson, G.D., \& Wilson, S.J. (2016b). Cognitionrelated brain networks underpin the symptoms of unipolar depression: Evidence from a systematic review. Neuroscience \& Biobehavioral Reviews, 61, 53-65.

Rayner, G., Jackson, G.D., \& Wilson, S.J. (2016). Two distinct symptom-based phenotypes of depression in epilepsy yield specific clinical and etiological insights. Epilepsy \& Behavior, 64, 336-344.

Rayner, G., Tailby, C., Wilson, S., \& Jackson, G. (2017). The spatiotemporal substrates of autobiographical recollection: Using event-related ICA to study cognitive networks in action. NeuroImage, 152, 237-248.

Rubin, D., \& Schulkind, M. (1997). The distribution of autobiographical memories across the lifespan. Memory \& 
Cognition, 25(6), 859-866. http://dx.doi.org/10.3758/ bf03211330

Salorio, C., White, D., Duntley, S., Uhles, M., \& Piccirillo, J. (2002). Learning, memory, and executive control in individuals with Obstructive Sleep Apnea Syndrome. Journal of Clinical \& Experimental Neuropsychology, 24(1), 93-100. doi:10.1076/ jcen.24.1.93.973

Strauss, E., Sherman, M.S., \& Spreen, O. (2006). A compendium of neuropsychological tests. New York: Oxford University Press.

Sumner, J.A., Griffith, J.W., \& Mineka, S. (2010). Overgeneral autobiographical memory as a predictor of the course of depression: A meta-analysis. Behaviour Research and Therapy, 48(7), 614-625.

Svaldi, J.J., \& Mackinger, H.F. (2003). Obstructive sleep apnoea syndrome: Autobiographical memory predicts the course of depressive affect after nCPAP therapy. Scandinavian Journal of Psychology, 44(1), 31-37.

Sweet, L.H., Jerskey, B.A., \& Aloia, M.S. (2010). Default network response to a working memory challenge after withdrawal of continuous positive airway pressure treatment for obstructive sleep apnea. Brain Imaging and Behavior, 4(2), 155-163.

Twigg, G.L., Papaioannou, I., Jackson, M.L., Ghiassi, R., Shaikh, Z., Jaye, J., ... Morrell, M.J. (2010). Obstructive sleep apnoea syndrome is associated with deficits in verbal but not visual memory. American Journal of Respiratory and Critical Care Medicine, 182, 98-103.

Viskontas, I., Mcandrews, M., \& Moscovitch, M. (2000). Remote episodic memory deficits in patients with unilateral temporal lobe epilepsy and excisions. The Journal of Neuroscience, 20(15), 5853-5857.

Wallace, A., \& Bucks, R. (2013). Memory and Obstructive Sleep Apnea: A meta-analysis. SLEEP, 36(2), 203-220. doi:10.5665/ sleep. 2374.9

Williams, J.M.G., \& Broadbent, K. (1986). Autobiographical memory in suicide attempters. Journal of Abnormal Psychology, 95(2), 144-149.

Williams, J.M.G., Barnhofer, T., Crane, C., Herman, D., Raes, F., Watkins, E., \& Dalgleish, T. (2007). Autobiographical memory specificity and emotional disorder. Psychological Bulletin, 133 (1), 122-148.

Young, T.D., Palta, M., Dempsey, J., Skatrud, J., Weber, S., \& Badr, S. (1993). The occurrence of sleep disordered breathing among middle-aged adults. New England Journal of Medicine, 328, 1230-1235.

Zigmond, A., \& Snaith, R. (1983). The Hospital Anxiety and Depression Scale. Acta Psychiatrica Scandinavica, 67(6), 361-370. 